\title{
PROCESOS DE ACOGIDA E INTEGRACIÓN PARA LA POBLACIÓN REFUGIADA EN BIZKAIA: ¿UNA REALIDAD A GEOMETRÍA VARIABLE?
}

\section{THE RECEPTION AND INTEGRATION PROCESS OF REFUGEES AND ASYLUM SEEKERS IN BIZKAIA: A REALITY WITH A VARIABLE GEOMETRY?}

Encarnación La Spina, Trinidad L. Vicente, Gorka Urrutia, Dolores Morondo*

Resumen: Los procesos de acogida e integración no solo son una realidad compleja y variable en la Unión Europea sino especialmente en contextos sociales determinados como las ciudades y las provincias. Estos deben afrontar los desafíos diarios de atención a las necesidades específicas de la población refugiada y garantizar la convivencia. Este trabajo recoge los resultados de una investigación interdisciplinar desarrollada sobre el grado de implementación de los procesos de acogida y de integración en Bizkaia atendiendo a las diferentes fases del Sistema de Acogida e Integración para personas solicitantes y beneficiarias de protección internacional. A tal

* Este trabajo se inscribe en el marco del Proyecto de la Diputación Foral de Bizkaia, convocatoria de proyectos Bizkailab, Bizkaia Solidarioa ref. 20160000210 Think Tank 2016: Derechos Humanos y Cooperación "La población refugiada en Bizkaia y su proceso de integración” así como en el proyecto de investigación "Análisis del proceso de acogida e integración de refugiados en España (2009-2016)" del Programa de Ayudas a Proyectos de investigación Aristos Campus Mundus, ref. ACM 2016_24. El artículo también se inscribe en el marco del Programa de Contratos Ramón y Cajal referencia RyC 2016-19465 financiado por el Ministerio de Economía, Industria y Competitividad y por el Fondo Social Europeo

Instituto de Derechos Humanos Pedro Arrupe. Universidad de Deusto 
propósito, se analiza la capacidad de los programas recientemente puestos en marcha, entre ellos especialmente el Programa Goihabe, para optimizar asi la coordinación y gestión integral de tales procesos en dicho territorio histórico.

Palabras claves: población refugiada; Bizkaia; integración; acogida; procesos.

Abstract: The processes of reception and integration are not only a complex and variable reality in the European Union but particularly in specific social contexts, such as cities and provinces. These must face the daily challenges of meeting the particular needs of refugees, ensuring daily coexistence. This paper presents the results of an interdisciplinary research on the degree of implementation of the reception and integration processes in Bizkaia, paying attention at the different phases of the Spanish Reception and Integration System for international protection applicants and beneficiaries. For that purpose, the capacity of recently launched programs, including the Goihabe Program, is analyzed in order to optimize the coordination and integral management of these processes in Bizkaia.

Key words: refugees; integration; reception; processes; Bizkaia.

\section{INTRODUCCIÓN}

Desde el inicio tanto de la crisis humanitaria como del sistema europeo común de asilo SECA, la mayoría de los debates teóricos y propuestas institucionales de reforma a nivel europeo y nacional se han centrado casi exclusivamente en dar solución al incremento de la llegada de población refugiada (European Asylum Support Office 2015). Sin embargo, los procesos de acogida e integración de las personas solicitantes de protección internacional no solo plantean múltiples interrogante y desafíos emergentes en términos cuantitativos (OCDE 2016, UNCHR 2013), sino que además requieren mejoras urgentes para garantizar una gestión integral y estratégica de las políticas europeas de inmigración y asilo (Agenda Europea de Migración y Asilo 2015, Global Compact on Refugees 2018).

Dentro de las disposiciones del acervo comunitario de inmigración y asilo (Peers et al., 2016), la hoja de ruta europea viene marcada por una armonización asimétrica de la Directiva 2013/33/UE sobre condiciones de acogida y la Directiva 2011/95/UE de reconocimiento 
en los Estados miembros (La Spina et al., 2017). Se aprecian diferencias no solo en las condiciones de acogida de las personas recién llegadas, en los procesos de protección temporal (como la reubicación o el reasentamiento de las personas que han entrado en el procedimiento de asilo por otro Estado), sino incluso más en la configuración del estatuto jurídico de la protección internacional que debería garantizar los procesos de inclusión (EMN 2015, Arango et al., 2016).

De hecho, las condiciones de acogida en la práctica europea han adquirido muy diferentes formas de un país a otro (EMN, 2013, EMN, 2015; Tsourdi, 2016, Offe, 2015); unas asimetrías que han hecho evidente la falta de garantías debidas en el acceso al sistema de asilo o la integración social (De Lucas, 2015; Naïr, 2016; Carrera et al., 2015), y que se han hecho más visibles, incluso, dentro de cada país, donde varían los tiempos, los mecanismos de coordinación de la red pública de acogida, los límites del marco competencial y los medios disponibles en los diferentes contextos locales específicos (OCDE, 2016; UNCHR, 2013).

En particular, este sería el contexto paradigmático y a geometría variable de los procesos de acogida e integración en España, por varias razones. En primer lugar, aunque las cifras de solicitantes de protección internacional continúan siendo muy bajas (solo el 1,15\% en comparación a la media europea) (EUROSTAT, 2017), cabe destacar la combinación de dos variables: de un lado, el progresivo incremento de las solicitudes de protección internacional (de 2.355 en 2012 hasta 15.770 en 2016) (ver gráfico 1), y de otro, el incumplimiento de los compromisos de reubicación temporal o reasentamiento de población refugiada proveniente de otros Estados europeos a España (Defensor del Pueblo, 2016: 90; Moraes y Romero, 2016).

En segundo lugar, dentro del marco jurídico, son remarcables los déficits del sistema de protección internacional español, la Ley 12/2009 reguladora del derecho de asilo y de la protección subsidiaria, modificada por la Ley 2/2014, de 25 de marzo (García Mahamut y Galparsoro, 2010), especialmente ante la ausencia de un desarrollo reglamentario y de transposición del acervo comunitario. Y, en tercer lugar, desde un plano institucional, es destacable la necesidad de reajuste de la estructura del sistema de acogida e integración (en adelante, $\mathrm{SAI}^{1}$ ) y de sus respectivas tres fases; esto es, de la red pública

1 Orden de 13 de enero de 1989 sobre centros de acogida a refugiados. «BOE» núm. 28, de 2 de febrero de 1989. 
GRÁFICO 1

\section{SOLICITUDES DE ASILO DE 2008 A 2016 EN ESPAÑA.} VALORES ABSOLUTOS

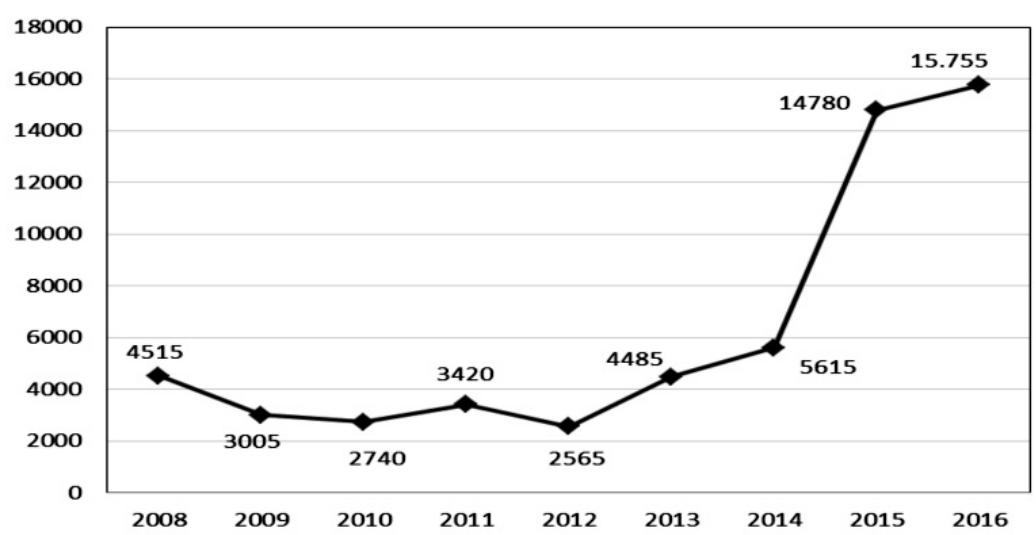

Fuente: elaboración propia con datos de Eurostat (2008-2016).

de acogida de personas solicitantes de protección internacional, que incluye los cuatro centros de acogida de personas refugiadas (CAR) dependientes del Ministerio de Interior (ubicados en Alcobendas y Vallecas en Madrid, en Sevilla y en Mislata en Valencia), así como los dos centros de estancia temporal de inmigrantes (CETI), también dependientes del Ministerio de Interior (localizados en Ceuta y Melilla). Y ello sin perjuicio de otros dispositivos de acogida dependientes del Ministerio de Empleo y Seguridad Social (MEYSS), gestionados por entidades y ONG especializadas en asilo como CEAR, ACCEM y Cruz Roja, que ofrecen atención básica, psicológica y escolarización de la población menor de edad, entre otros servicios de apoyo ${ }^{2}$.

En la implementación práctica y multinivel del SAI es, sin embargo, donde se observan ciertas disfuncionalidades y velocidades en los diferentes contextos locales, siendo precisamente ahí donde los procesos de acogida e integración están llamados a convertirse

2 Según la información facilitada por el Defensor del Pueblo (2016), en el año 2015 el número de plazas gestionadas por las organizaciones no gubernamentales ascendía a 502, pasando a 2.000 en el año 2016. 
en un ámbito prioritario de acción y de investigación comparada (La Spina et al., 2017, p. 122-128; Scholten \& Penninx, 2015). Y ello por ser, en definitiva, quienes deben afrontar los desafíos diarios, así como los conflictos de convivencia que pueden originarse, dada la proyección y múltiples dimensiones de la integración a corto y largo plazo (Pascouau, 2014; European Parliament, 2016).

La complicada coordinación entre las diferentes administraciones competentes es una cuestión relevante para el desarrollo de las políticas de la acogida y posterior proceso de integración de las personas solicitantes de asilo y refugiadas en tales contextos específicos. No en vano, han sido varios los informes y estudios que han dado cuenta de los problemas de ajustes de la concepción lineal y acumulativa del SAI en diferentes comunidades autónomas (CC.AA.), especialmente en el tránsito de la fase de acogida a la de integración, así como una vez finalizadas las ayudas económicas del SAI (Iglesias et al., 2018; Iglesias y Estrada, 2018).

\section{GRÁFICO 2}

EVOLUCIÓN HISTÓRICA DE LAS SOLICITUDES DE PROTECCIÓN INTERNACIONAL EN EUSKADI Y EN BIZKAIA. AÑOS 2009-2015. VALORES ABSOLUTOS

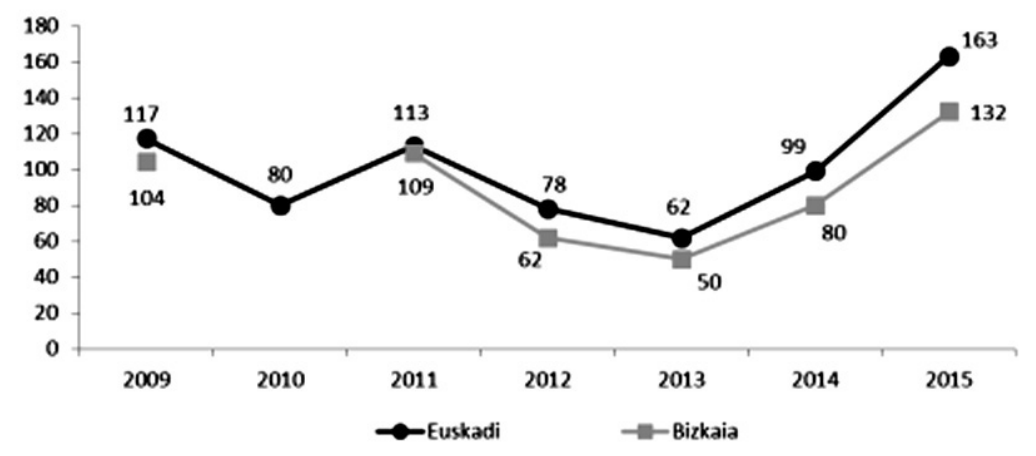

Fuente: elaboración propia a partir de datos del Ministerio del Interior (2009-2015).

Nota: no se dispone del dato de solicitudes de protección internacional en Bizkaia en el año 2010.

Este diagnóstico de los problemas de ajustes del SAI y su impacto en los procesos de inserción social resulta también significativo en Euskadi, y más concretamente en Bizkaia, un territorio que ha experimentado un notable incremento en el volumen de solicitudes 
de asilo tramitadas (Gráfico 2), y donde la necesidad de reforzar la acogida y la integración ha tenido su reflejo, al igual que en otras CC. AA. ${ }^{3}$ en las crecientes movilizaciones y sensibilización social e institucional a la hora de reclamar mejoras en los mecanismos de acogida e integración conveniados.

El presente trabajo se inserta en este contexto específico y recoge los resultados obtenidos tras el análisis, en primer lugar, del grado de implementación de los procesos de acogida y de integración de las personas solicitantes de protección internacional en Bizkaia, atendiendo a las diferentes fases del Sistema de Acogida e Integración a ellas dirigido. Y, en segundo lugar, trata de evaluar la capacidad de los programas recientemente puestos en marcha, entre ellos especialmente el Programa Goihabe, para optimizar la coordinación y gestión de tales procesos en dicho territorio histórico.

\section{Marco teórico}

Los procesos de integración de las personas migrantes son un fenómeno multidimensional y complejo (Giménez, 2003) que puede variar en tiempo y espacio según el país, las circunstancias histórico-políticas y los diferentes modelos de integración (Joppke, 2007). En todo caso, la noción de integración parece ir asociada a una connotación positiva que ya ha comenzado a ser cuestionada, al igual que la ambigüedad conceptual y la confusión terminológica que parecen acompañar a dicho concepto (Blanco, 1990; Pumares, 1998; Koopmans, 2010). Aun así, y a sabiendas de que no existe un consenso y de las dificultades que ello entraña, en este artículo definiremos la integración como un fenómeno multidimensional y bidireccional condicionado por diferentes factores socioeconómicos, sociolaborales, políticos, residenciales, culturales y, de igualdad de derechos, que implican un proceso de adaptación mutuo tanto de la comunidad a integrar como de la sociedad de destino.

La integración de la población refugiada, como solución duradera y a largo plazo ante el actual escenario de crisis humanitaria

3 Andalucía, Aragón, Asturias, Canarias, Cantabria, Castilla-La Mancha, Cataluña, Comunitat Valenciana, Extremadura, Islas Baleares, País Vasco y Navarra han mostrado su disposición a colaborar en la acogida y la integración social de las personas refugiadas así como una distribución adecuada. 
permanente en Europa, ha ampliado los horizontes del ámbito de reflexión crítica sobre los modelos teóricos existentes y ha retomado el interés de la doctrina sobre aspectos menos estudiados de las migraciones forzadas (UNCHR, 2013; Fielden, 2008). Básicamente porque hasta ahora las políticas de integración estaban pensadas prioritariamente para las personas nacionales de terceros estados residentes en el territorio sin tener en cuenta las diferentes situaciones de protección internacional, como parte de este grupo a integrar. Y, también por la falta de evidencias empíricas y datos cuantitativos sobre la integración social de las personas refugiadas frente a la abundante investigación cualitativa sobre la integración de personas inmigrantes (EP, 2015; AIDA, 2016). En definitiva, estos aspectos más deficitarios dan paso a una investigación emergente más centrada en teorizar y fijar las particularidades de la primera fase de acogida de la población refugiada, las diferencias entre los patrones de integración a medio y largo plazo de las personas inmigrantes o refugiadas y, al mismo tiempo, más preocupada en profundizar en la valoración de la necesidad de implementar o no prácticas específicas de cara a lograr la inclusión de la población refugiada, o de su inclusión únicamente en programas de integración más genéricos (Iglesias et al., 2018; Bauböck \&Tripkovic, 2017).

\section{Metodología}

Este artículo sobre la implementación de los procesos de acogida e integración en Bizkaia se nutre de los resultados obtenidos de una primera investigación llevada a cabo en este contexto específico por un equipo del Instituto de Derechos Humanos Pedro Arrupe, en el marco del proyecto Bizkailab: "La población refugiada en Bizkaia y su proceso de integración". Una investigación interdisciplinar que, dado su carácter exploratorio, ha optado por una metodología de carácter cualitativo, basada concretamente en entrevistas en profundidad semiestructuradas, tanto a personas solicitantes de protección internacional residentes en Bizkaia como a personas expertas informantes claves. Por una parte, se ha entrevistado a ocho personas residentes en este territorio que han solicitado protección internacional, con el objeto de conocer sus motivos, sus propias experiencias, su vivencia migratoria de carácter familiar y sus valoraciones y expectativas del proceso de solicitud de asilo que 
han vivido o están viviendo desde el punto de vista de su situación familiar, educativa, social, laboral y económica. Y, por otra parte, esta metodología cualitativa ha sido completada con entrevistas en profundidad mantenidas con catorce personas expertas, conocedoras por su propio desarrollo profesional de la realidad de las personas solicitantes de protección internacional. Concretamente se ha entrevistado a seis representantes de cuatro organizaciones encargadas de la acogida y/o integración de personas solicitantes de asilo en Bizkaia, a tres personas técnicas de inmigración en el ámbito municipal, a una representante de la Asociación de Municipios Vascos Eudel, a dos personas del Departamento de Empleo y Políticas Sociales y a otra del Departamento de Educación del Gobierno Vasco, y a una persona del Departamento de Empleo, Inclusión Social e Igualdad de la Diputación Foral de Bizkaia. Todas estas entrevistas en profundidad fueron realizadas a lo largo del mes de noviembre y diciembre de 2016.

\section{ANÁLISIS DE LOS PROCESOS DE ACOGIDA E INTEGRACIÓN EN BIZKAIA}

\subsection{La acogida de la población refugiada en Bizkaia}

El objetivo del SAI es prestar alojamiento, manutención, asistencia psicosocial urgente y primaria y facilitar el acceso a los servicios sociales encaminados a favorecer la convivencia e integración de las personas que solicitan protección internacional y carezcan de medios económicos para atender sus necesidades o las de su familia. Las personas beneficiarias de estas ayudas o programas especiales deben ser residentes en un centro de acogida (o bien estar fuera pero siguiendo un programa de atención iniciado como residente), carecer de recursos económicos o no tener cobertura de otros servicios sociales generales $\mathrm{u}$ organismos privados ${ }^{4}$.

El proceso de acogida en Bizkaia comparte elementos comunes y sinergias con la llamada fase de acogida del SAI, pese a no tener en su territorio un establecimiento público (Amnistía Internacional, 2016; CEAR, 2016). La puesta en marcha de un itinerario de

4 Real Decreto 865/2006, de 14 de julio, «BOE» núm. 168, de 15 de julio de 2006 . 
acogida altamente centralizado implica, sin embargo, cierto grado de complejidad que, en determinados momentos, puede generar confusión o solapamientos en relación con los servicios que deben prestar los distintos agentes implicados, incluyendo el Gobierno español, el Gobierno vasco y otras administraciones públicas de ámbito local, y las distintas ONGs (implicadas en el sistema tanto a nivel estatal como local). Ello plantea algunas dificultades a la hora de gestionar la acogida inmediata y la ubicación de la persona solicitante de protección, ya que en ocasiones esto implica una acogida en dos momentos distintos, lo que también depende o requiere ajustarse a las condiciones a nivel local de las entidades encargadas de esa gestión (Cruz Roja, CEAR y ACCEM).

En la primera parte nosotros simplemente le decimos que si no ha solicitado en Policía la cita, que vaya a Policía y que luego vaya a CEAR. (...) A nosotros solo nos va a venir gente dada, es decir, que el Ministerio vaya a decir: "chicos, tenéis una plaza reservada". Entonces nosotros, con la entidad que deriva, tramitamos el viaje, acordamos qué día llega para poder ir a recibirle y le ingresamos en un centro nuestro. Nosotros en Bizkaia tenemos 10 pisos, 56 plazas. (...). Entonces en dependencia de si hay una plaza libre o no, el Ministerio decide: pues a Bilbao, a Donostia, o a donde sea. (Representante de ONG encargada de la acogida y/o integración de solicitantes de asilo en Bizkaia)

En la llegada de personas solicitantes de protección internacional, y en particular con relación a aquellas personas acogidas en Bizkaia, es necesario distinguir entre quienes llegan a este territorio derivadas de otras CC. AA. y quienes inician el proceso de solicitud en este territorio. El lugar de ubicación de la persona solicitante de protección dependerá de varios factores, y el hecho de disponer de una red de apoyo puede incidir en la determinación de dicho lugar. En el caso de Bizkaia, se puede observar que hay personas solicitantes de protección con diversos perfiles: aquellas que llegan (o solicitan la protección) por primera vez a Bizkaia, aquellas que tienen redes de apoyo en este territorio y aquellas personas que son derivadas de otras zonas de ámbito estatal para su ubicación en algún centro con espacio libre.

El papel que juegan las entidades sociales para esta cobertura se presenta como un elemento central. El hecho de contar con una red de apoyo familiar puede suponer un elemento positivo, entre 
otras razones porque puede determinar el lugar de ubicación de estas personas. Así, quienes cuenten con esa red tendrán mayores opciones de ser ubicados en el lugar en donde dispongan de esa red y, consecuentemente, será más fácil la integración y acogida. Sin embargo, este aspecto aparentemente positivo no siempre funciona de este modo. En determinadas ocasiones, hacer uso de la cobertura familiar (o de otras redes de apoyo), puede ir en contra de las personas que han iniciado su itinerario en el SAI, ya que este hecho puede provocar su salida del proceso y sistema del MEYSS, tal y como refleja el siguiente testimonio:

Hasta hace unos meses si rechazaban plaza en centro quedaban fuera del programa. (...) Es ridículo que vaya a un centro de acogida y ocupe una plaza que igual es necesaria para una persona que no tiene la red de apoyo. Entonces, ahora por eso ha cambiado un poquito. (...) Hoy en día, rechacen o no, siguen en el programa. (Representante de ONG encargada de la acogida y/o integración de solicitantes de asilo en Bizkaia)

Por otra parte, hay que destacar también que, en ausencia de dicha red familiar o de apoyo, las personas solicitantes de asilo se enfrentan a dificultades para cubrir sus necesidades básicas durante el tiempo que transcurre desde su llegada hasta su ingreso en el SAI. Una situación que, en el caso particular de Bizkaia, trata de solventarse, así sea de forma puntual y como un apoyo adicional al sistema existente, a través de algunos equipamientos municipales destinados a albergar a personas en general, y a solicitantes de asilo en particular. El proceso en el que se encuentran las personas solicitantes de protección tiende a bascular entre el proceso formal de acogida y otros vinculados a los procesos migratorios de carácter más general (la dificultad del aprendizaje del idioma, la búsqueda de empleo, la búsqueda de vivienda, etc.). En este caso específico de las personas solicitantes de asilo, se han hecho explícitas algunas dificultades adicionales propias de un proceso de estas características, como son:

[¿Ayuda médica y psicológica?] No. Nada. Yo creo que era lo más necesario para nosotros en ese momento. [...] Creo que estas personas antes que alimento, o su primer alimento, es tener una psicóloga; alguien que le saque todo el dolor que tiene, que pueda hablar con alguien. (Mujer, 34 años, kurda, estudios secundarios, con estatuto de refugiada) 
[Dificultades por la lengua] Los primeros tres meses fue muy difícil. Cuando hablaba muchas personas no hablan inglés, solo castellano, pero yo no entendía qué pasaba. (...) Ahora poco a poco, entiendo. (Mujer, 35 años, pakistaní, maestra, solicitante de asilo)

Paralelamente al proceso de llegada, y desde una perspectiva temporal, se inicia el proceso administrativo de la solicitud de protección, que es lo que da acceso al SAI y ofrece el soporte durante, al menos, los primeros seis meses de estancia en el país. El punto de arranque de esta situación se produce con la tramitación de inicio del proceso, con la asistencia a la comisaria de policía para llevar a cabo la entrevista; unos trámites que suelen ser relativamente ágiles y en los que el apoyo de las entidades sociales suele ser fundamental. Esta agilidad, sin embargo, choca con la extensión temporal del itinerario que comienzan a recorrer una vez han acudido a la comisaria e iniciado los trámites. Son pocas las personas solicitantes de protección que reciben una comunicación rápida. Adicionalmente, otros obstáculos agravan la angustia y preocupación de las personas solicitantes de asilo, como son: las dificultades para encontrar trabajo, la sensación de estar pendiente de un proceso desconocido, la falta de libertad, el temor a que la solicitud sea denegada...:

Es un poco difícil para encontrar trabajo. [...] Tengo el permiso y también estoy buscando en varios lugares. Por ejemplo, en los hoteles, porque tengo experiencia en esos lugares. Y en bares, y cafeterías; como camarero en pisos, cosas como éstas. Estoy buscando, pero no es fácil. (Hombre, 45 años, yemení, estudios universitarios, solicitante de asilo)

Llevo casi un año y medio [desde que hizo la solicitud]. He renovado la tarjeta roja tres veces. (Hombre, 45 años, yemení, estudios universitarios, solicitante de asilo)

Enmarcadas en el proceso de huida y salida de sus lugares de origen, estas circunstancias no contribuyen a facilitar ni el propio proceso de llegada y de solicitud de protección ni, por extensión, el de sus familiares directos (La Spina 2017):

En estos países tienes que salir de forma ilegal, para personas como nosotros. Entonces, como mujer no tenía pasaporte, porque para tener pasaporte en Irán las mujeres menores de 40 años tienen que tener permiso del padre, hermano o marido. [...] 
Para escolarizar al niño el problema lo tuve en Euskadi. [...] En Irán los hijos no llevan el apellido de la madre y me pedían libro de familia, pero nosotros no teníamos libro de familia. (Mujer, 34 años, kurda, estudios secundarios, con estatuto de refugiada)

La mayoría de las personas inician este proceso al llegar al país, sobre todo aquellas que proceden de contextos con situaciones de conflictos armados directos. Ahora bien, esta falta de información precisa sobre el proceso es mucho más llamativa en el caso de las personas que solicitan el asilo después de un tiempo de estancia en el país. Este es otro perfil de personas que solicitan la protección que emerge de las entrevistas, personas que han inmigrado hace tiempo (incluso varios años) e inician el proceso de solicitud después de ese tiempo. A la decisión de iniciar el proceso llegan a través de conversaciones con otras personas inmigrantes o cuando toman contacto con entidades sociales que les plantean esta posibilidad, tras conocer su realidad:

Personas que han venido y se han quedado en situación administrativa irregular y un día, hablando con un técnico le pueden contar su caso y al de seis meses o un año, pueda decir: "oye, pues esto puede ser un caso de asilo". Y se deriva a CEAR. (Representante de ONG encargada de la acogida y/o integración de solicitantes de asilo en Bizkaia)

El último de los elementos que concurre de manera paralela a los dos anteriores es el del alojamiento, esto es, el de la ubicación física de las personas que han solicitado la protección internacional. El SAI contempla un proceso de acogida para los primeros seis meses en pisos gestionados por las entidades sociales vinculadas a los procesos de protección (CEAR, ACCEM y Cruz Roja, entre otras). El número de plazas que se gestionan en Bizkaia para este primer periodo está determinado por el propio SAI y las dos entidades implantadas en Bizkaia (CEAR y Cruz Roja), cuestión esta que condiciona la disponibilidad de alojamiento para esta primera fase.

El impacto de la crisis migratoria y las medidas activadas durante estos últimos tiempos ha hecho que se dé un aumento de plazas, tanto desde la administración pública como desde las entidades sociales, y que se planteen alternativas para dar una mejor cobertura a las necesidades que van surgiendo, tal y como se concreta en las siguientes líneas: 
De tener en septiembre 10 plazas, ahora mismo tenemos 61 plazas en Bizkaia, tenemos 42 en Álava, y 16 en Gipuzkoa. Entonces nosotras, por ejemplo, nos hemos multiplicado por 12. Cruz Roja en Euskadi no disponía de ninguna plaza de acogida, entonces todo lo que tiene en Vitoria, Gipuzkoa y Bilbao, todo es nuevo. (Representante de ONG encargada de la acogida y/o integración de solicitantes de asilo en Bizkaia)

En lo que respecta a la cobertura de las necesidades básicas de las personas solicitantes de asilo, las entidades sociales son las que asumen la competencia de esa gestión durante el tiempo de estancia en estos pisos:

En esos seis meses que está bajo nuestra tutela, que aprenda y se forme lo más rápido posible para que cuando le den ese permiso de trabajo pueda optar ya a un trabajo con una formación y con unas prácticas o con una pequeña experiencia. Mientras está bajo nuestra tutela tiene todas las necesidades básicas cubiertas: vestuario, alimentación, formación, todo lo que es necesario. (Representante de ONG encargada de la acogida y/o integración de solicitantes de asilo en Bizkaia)

\subsection{La integración de la población refugiada en Bizkaia}

El papel de las ciudades o las Comunidades Autonómas en la acogida e integración de la población refugiada no está hoy en día suficientemente reconocido (artículo 149.1.2 CE) o promovido (Disposición Adicional Cuarta "Cooperación con otras Administraciones Públicas” de la Ley 12/2009), incluso teniendo en cuenta las competencias asumidas en ámbitos que afectan directamente la integración social de las personas inmigrantes y, por ende, de las solicitantes de asilo, refugiadas o beneficiarias de protección subsidiaria. Actualmente, en el ámbito de las políticas de integración, sólo seis comunidades autónomas (Aragón, Asturias, Castilla y León, Cataluña, C. Valenciana y País Vasco) tienen algún tipo de plan en vigor dirigido a las personas migrantes, inclusive población refugiada, para su integración y convivencia. Especialmente, la Comunidad de Madrid y Cataluña (Foro para la Integración Social, 2015), siendo las que reciben mayor volumen de personas solicitantes, han previsto planes específicos de atención 
para las personas refugiadas a nivel local o autonómico ${ }^{5}$. Las personas solicitantes de protección internacional, durante la $1^{\circ}$ fase de acogida y frecuentemente durante la $2^{\circ}$ fase, denominada de integración, van a contar con un importante apoyo por parte del Estado para cubrir sus necesidades básicas. Un periodo que varía entre los 6 y los 18 meses, pudiendo llegar en algunos casos hasta los 24 meses. Sin embargo, hasta la resolución del expediente de asilo, los derechos vinculados a la acogida e integración tratan de cubrir temporalmente necesidades básicas como las prestaciones sociales, la asistencia sanitaria de urgencia o bien la asistencia jurídica gratuita e intérprete en el momento de la solicitud de asilo y la tramitación del expediente.

Las personas solicitantes de asilo que ven su solicitud denegada - la gran mayoría tanto en el Estado español como en la CAPV-, van a experimentar un cambio radical de estatus, perdiendo por ello todos los apoyos estatales de los que disponían hasta ese momento para pasar a una situación de irregularidad administrativa.

Incluso aquellas personas que, gracias a disponer de una autorización temporal de trabajo, hubieran conseguido un empleo que les proporcionara los medios básicos de vida, van a encontrarse con una difícil situación laboral, derivada de la pérdida de dicho permiso de trabajo. Un cambio que, sin duda, les va a enfrentar con la realidad de la exclusión, ya que frecuentemente tampoco van a cumplir las condiciones necesarias para acceder a ayudas sociales, como son la Renta de Garantía de Ingresos (RGI) (que requiere tres años de empadronamiento en la CAPV). Esta situación contrasta con la de las personas refugiadas con estatuto reconocido y aquellas beneficiarias de protección subsidiaria, que acceden a las prestaciones sociales o a la asistencia sanitaria en términos de equiparación de condiciones con la población española, mientras que el estatuto jurídico de quienes no cuentan todavía con un estatuto de protección internacional es asimilable al de las personas inmigrantes residentes.

Pero también las personas a las que se les reconoce protección internacional van a constatar cómo las ayudas otorgadas por el Ministerio se reducen paulatinamente en la fase de integración

5 Plan de Atención integral a los refugiados de la Comunidad de Madrid de 8 de septiembre de 2015 y Plan de protección internacional en Cataluña de 28 de diciembre de 2014. 
y, sobre todo, de autonomía, de modo que van a ser ellas mismas quienes van a tener que buscar sus propios medios de subsistencia y, en caso de no conseguirlos, acudir a la atención pública destinada a personas en situación de vulnerabilidad. Una atención que ni en el caso de las personas inmigrantes en situación irregular ni en el de las personas con estatuto de refugiadas se plantea de forma específica para ellas, sino que se enmarca en las medidas proporcionadas a la ciudadanía en su conjunto.

A continuación, como resultado de la investigación realizada, se seleccionarán algunos de los ámbitos concretos de intervención social que presentan más dificultades de coordinación y que son imprescindibles para la integración de la población refugiada en Bizkaia.

\section{Acceso al empleo y cobertura de necesidades para promover la inserción socio-laboral}

De acuerdo con los artículos 17, 18 y 19 de la Convención de Ginebra sobre el Estatuto de refugiado, un aspecto clave de la inserción socio-laboral es el derecho al trabajo remunerado, ya sea por cuenta ajena o propia, garantizando "el trato más favorable posible y en ningún caso menos favorable que el concedido en las mismas circunstancias generalmente a la población extranjera" (Arnold-Fernández y Stewart, 2014). La persona solicitante de protección internacional está autorizada a trabajar a partir de los seis meses desde la formalización de su solicitud, tras solicitar la renovación de su documentación, en la que figurará el plazo de validez del documento que se le expida.

La concesión de las autorizaciones de residencia y trabajo temporal es una competencia exclusiva del Estado. Esta autorización (llamada tarjeta roja) es un título habilitante de residencia y trabajo equivalente a los permisos de larga duración. Aunque, como es obvio, su mera posesión no supone la superación de los grandes obstáculos en la inserción laboral que han de afrontar las personas inmigrantes, en general. Además comporta elementos de incerteza, ya que, como se ha indicado anteriormente, la duración del proceso de solicitud es indeterminada (pudiendo durar de seis meses a año y medio, lo que tiene un efecto negativo en la demanda y la oferta de empleo) (CEAR, 2016; Amnistía internacional, 2016). Y, por otra parte, la denegación de la solicitud, que podría llegar en cualquier momento, implicaría la 
pérdida de dicho permiso de trabajo, siendo el empleo sumergido la única opción laboral para las personas en dicha situación de acuerdo a la legislación de extranjería, con las consiguientes implicaciones personales y familiares que ello conlleva:

Con la segunda tarjeta ya tienen el permiso para trabajar. Pero si en mitad de ese camino ellos ya han conseguido trabajo, están cotizando, y de repente hay una denegación, automáticamente, pues eso, dejan de cotizar. Dejan de estar en una situación administrativa regular para pasar a irregular. Entonces el contrato cesa. Se cesa también todo el tema de las cotizaciones. (Representante de ONG encargada de la acogida y/o integración de solicitantes de asilo en Bizkaia)

También es preciso tener en cuenta que dicho permiso laboral no asegura una incorporación rápida y favorable en el mercado de trabajo, máxime en la actual coyuntura socioeconómica de crisis y de empleo, en la que se han visto especialmente afectados los segmentos más bajos del mercado laboral. Unos nichos laborales - como la construcción- que son precisamente los ocupados por la población inmigrante tanto en España en general como en la CAPV en particular. En este contexto, tampoco sorprende que se considere más fácil la incorporación de aquellos colectivos de inmigrantes mejor aceptados por parte de la sociedad vasca (como los latinoamericanos frente a los magrebíes, por ejemplo), o de las mujeres en comparación con los hombres (aunque sea en sectores con una condicionales laborales precarias, como las ofrecidas por el sector del servicio doméstico y de cuidados).

Generalmente, el no dominio de alguno de los dos idiomas de la CAPV se considera una seria dificultad en la incorporación laboral. Pero no es la única, ya que a esta se unen otras como, por ejemplo, los reducidos ámbitos de empleo destinados a la población inmigrante en general y solicitante de asilo en particular. Así, como se indicaba antes, una gran mayoría de mujeres procedentes de otros países va a ver reducidas sus ofertas de empleo al trabajo doméstico y de cuidados, y ello con independencia de su formación previa, mostrando por ello un gran descontento con este "encasillamiento" laboral, así como con las condiciones ofrecidas por estos trabajos: inestabilidad, trabajo a tiempo parcial, bajos salarios, etc.

Una reducida oferta laboral que va a hacer que algunas personas opten por otras alternativas laborales, así las tengan que desarrollar 
dentro de la economía sumergida. Entre las personas con un nivel educativo más alto, la aspiración por lograr un empleo más acorde con su formación, e incluso con su experiencia laboral previa, permanece, y a ello van a dedicar importantes esfuerzos. Pero no por ello se puede renunciar a una incorporación laboral en estos sectores más bajos; lo que, a su vez, va a suponer un importante freno al logro del objetivo anterior, al ser difícil compatibilizar el desempeño de dicho trabajo remunerado con los tiempos y esfuerzos económicos que requieren los procesos de formación y de convalidación de títulos que les permitirían avanzar en este sentido.

Por último, es importante destacar la importancia del capital social, de la red de contactos de estas personas en la búsqueda y consecución de un empleo. En la mayoría de los casos, dada la naturaleza de los puestos de trabajo ocupados, no es la intermediación de Lanbide (Agencia Vasca de Empleo) o de las agencias de colocación la que juega un papel central en la consecución de un empleo, sino las recomendaciones, la información o los contactos de amistades y/o familiares.

\section{Formación profesional y homologación de títulos}

Muchas de las personas solicitantes de asilo entrevistadas, con independencia del estado de su solicitud, han iniciado alguna actividad de formación con el objeto de mejorar sus oportunidades en el mercado laboral vasco. Un objetivo para el que es determinante la inscripción en los Servicios Públicos de Empleo. A través de esta inscripción, que debe ser renovada, se tiene la posibilidad de acceder a formación ocupacional subvencionada y, una vez que se disponga de autorización de trabajo, es posible participar de su servicio de intermediación con empresas que ofertan empleo, así como solicitar la homologación de los estudios académicos realizados en su país origen ante el Ministerio de Educación.

Esta formación profesional, ofrecida por Lanbide y por otras entidades sociales, suele estar dirigida a mejorar la empleabilidad de este colectivo teniendo más en cuenta los puestos ofrecidos por dicho mercado de trabajo que la formación previa de las personas que los emprenden. La realización de este tipo de formación profesional requiere de un cierto nivel de conocimiento del español; lengua en la que se imparte. Pero también contribuye a su perfeccionamiento. Por otra parte, las personas solicitantes de asilo disponen de más tiempo 
para realizar estos cursos durante la etapa de acogida, pero durante la fase de integración o de autonomía, o si han visto denegada su solicitud, se enfrentan a mayores dificultades para poder seguir esta formación, al tener que compaginarla con el desempeño o con la búsqueda de trabajo remunerado.

De igual manera, la posibilidad de finalización de la carrera por parte de aquellas personas que tuvieron que abandonar su país cuando apenas les quedaba algún curso o algunas asignaturas para hacerlo, constituye un gran reto para el sistema educativo en general y para el sistema universitario en particular. Una medida que mejoraría la posición en el mercado de trabajo de estas personas, proporcionándoles seguramente un empleo mucho más satisfactorio y ofreciendo, al mismo tiempo, una mayor aportación al conjunto de la sociedad.

\section{Conocimiento de los idiomas oficiales en la CAPV}

Un elemento clave para la integración es, sin duda, el conocimiento de la lengua o lenguas oficiales de la sociedad de recepción. Por ello, la mayoría de las personas solicitantes de asilo que no conocen el español suele comenzar a estudiar este idioma durante la fase de acogida. Esto no obstante, el aprendizaje de dicha lengua suele precisar de un tiempo superior al que abarca este periodo, e incluso superior a la etapa durante la cual se extiende la protección otorgada por el Ministerio. De hecho, tanto las personas a las que se aprueba la solicitud de protección como aquéllas a las que se les deniega sienten la necesidad de seguir asistiendo a clases de español. La asistencia a clases de euskera es, en cambio, mucho más reducida y eventualmente se inicia más tardíamente.

De las necesidades de formación lingüística se ocupa principalmente la EPA (Educación Permanente de Adultos), un programa desarrollado por el Departamento de Educación del Gobierno Vasco, que ofrece semanalmente clases gratuitas de español, de euskera y de educación básica para personas adultas, con independencia de su origen o de su situación jurídica. Una oferta que es completada también por parte de las Escuelas Oficiales de Idiomas, también dependientes del citado Departamento de Educación, aunque en este caso resulta difícil la obtención de una plaza para quienes no alcanzan a solicitarla en el mes de septiembre. La oferta de la EPA y de las Escuelas Oficiales de idiomas, considerada insuficiente por 
algunos agentes sociales, es complementada, asimismo, por otras entidades sociales, como son Cruz Roja, Fundación Ellacuría o Ítaca Escolapios:

Hemos empezado también algo de refuerzo del idioma, porque nos da la impresión y vamos viendo que la gente que tiene perfil de asilado y que está recibiendo en un programa de protección algunas clases de castellano, pues necesita todavía refuerzos, porque esos programas son cortos y en seis meses, o en nueve meses, digamos que la gente no controla bien la lengua. (Representante de ONG encargada de la acogida y/o integración de solicitantes de asilo en Bizkaia)

La principal limitación que plantean estas clases de español es la relativa a las horas docentes ofrecidas; valoradas a menudo como insuficientes dada la urgencia por manejar el idioma. Sin embargo, también se apunta la dificultad de compatibilizar los tiempos de estudio y de trabajo, cuando se consigue el tan ansiado empleo. Y, por otra parte, se señala que en estas clases muchas veces se encuentra a más gente extranjera que comparte su mismo idioma, siendo más sencillo recurrir al mismo que conocer a personas autóctonas con las que poner en práctica los nuevos conocimientos idiomáticos adquiridos. El propio Gobierno Vasco se muestra conocedor de esta situación, y señala que podrían estudiarse algunas medidas alternativas desde las propias EPAs o, incluso, desde Lanbide. Aunque apunta, asimismo, que sería preciso que las demandas de nuevos servicios se realizaran de forma concreta, para poder dar una mejor respuesta a las mismas. En todo caso, el no dominio del idioma es percibido como una barrera a la incorporación social en general y a la inserción laboral en particular.

\section{Acceso a escolarización de menores, vivienda y servicios sociales}

Uno de los efectos atribuidos a la concesión del estatuto de protección internacional es que el acceso a la educación, a la asistencia sanitaria, a la vivienda, a la asistencia social y a los servicios sociales, o a los derechos reconocidos por la legislación aplicable a las personas víctimas de violencia de género, debe garantizarse en las mismas condiciones que a la población española (Fernández, 2015). En el caso de personas solicitantes de protección internacional a las que no se les ha reconocido el estatuto, el acceso continuado a 
tales derechos sociales básicos va a quedar condicionado al registro en el Ayuntamiento del lugar de residencia (empadronamiento) y a su consiguiente renovación, cada dos años, si no se tiene residencia permanente.

\section{Escolarización de menores}

El principal trámite es el empadronamiento de toda la unidad familiar y la solicitud de la plaza en los servicios de escolarización correspondientes, ya que la escolarización es obligatoria para los niños y niñas entre los 6 y los 16 años. Cada Comunidad Autónoma es responsable de los servicios de educación en su territorio. La escolarización de menores en la CAPV es un derecho garantizado, con independencia de su origen o de su situación administrativa (Vicente, 2008). Es por ello que el Departamento de Educación del Gobierno Vasco no estima oportuno diseñar ninguna actuación específica en este sentido:

Protocolos de acogida para alumnado refugiado, pues no tenemos. Tenemos un protocolo muy potente y un montaje muy potente para alumnado, vamos a decir, inmigrante (...) Porque la problemática de un niño refugiado es la misma que la de un inmigrante. Es un problema idiomático, es un problema cultural. (Representante del Departamento de Educación, Gobierno Vasco)

Dicho esto, de las entrevistas mantenidas se desprende que dicho proceso de escolarización no siempre es sencillo, debido a los trámites burocráticos exigidos para ello (y que frecuentemente no tienen en cuenta la realidad de las personas que llegan y solicitan asilo):

Para escolarizar al niño el problema lo tuve en Euskadi. (...) Cuando fui a empadronarme ahí, había problemas. Me han dicho que no iban a empadronar a mi hijo. En Irán los hijos no llevan el apellido de la madre y me pedían libro de familia, pero nosotros no teníamos libro de familia. (...) Y yo lo que necesitaba era empadronar al niño para poder escolarizarlo. (...) Estuvo mi hijo tres meses sin colegio. (Mujer, 34 años, kurda, estudios secundarios, con estatuto de refugiada)

En este sentido cabe destacar que, como se ha apuntado en varias entrevistas, hasta ahora el personal municipal no ha recibido 
una formación específica en materia de asilo, por lo que desconoce su particular realidad. Ahora bien, sí parece que se está planteando llenar esta laguna, con una formación especialmente dirigida al personal del área de servicios sociales:

Vamos a formar a todos los ayuntamientos, los responsables políticos y técnicos, en lo que es asilo, refugio y un poco en lo que es la multiculturalidad. (Representante del Departamento de Empleo y Políticas Sociales, Gobierno Vasco)

Yo creo que se está planteando ahora una formación para Servicios Sociales. Pero hasta ahora no ha habido. (Representante de Eudel)

Desde el punto de vista educativo, una vez en los centros escolares vascos, este alumnado también ha de enfrentar otros problemas, especialmente cuando llegan a edades más avanzadas. Entre ellos, el más frecuentemente apuntado es el vinculado al aprendizaje de, al menos, uno de los idiomas oficiales de la CAPV, cuando no de los dos. Un aspecto que ha sido tenido en cuenta por el Departamento de Educación y para el que se ha creado la figura del profesorado de refuerzo lingüístico, aunque su presencia por un tiempo limitado - dos cursos, sin posibilidad de alargamiento- ya se ha mostrado insuficiente en muchos casos y con claras implicaciones en el fracaso escolar de este alumnado de origen extranjero.

Pero además de éste, existen otros obstáculos, como la no elección de red educativa y/o de centro escolar por parte del alumnado que llega fuera del periodo ordinario de matriculación, el cambio de red educativa y de centro escolar cuando es posible hacerlo, o el coste económico de libros, material y comedor. En cambio, donde sí parece haberse registrado un avance positivo es en la educación infantil no obligatoria, en la ofrecida por las Haurreskolas o escuelas infantiles, que tienen abiertas sus puertas de forma gratuita para los niños y las niñas solicitantes de asilo o que ya disfrutan de protección internacional.

\section{Vivienda y servicios sociales}

El entorno residencial y el acceso al espacio público de prestaciones son dimensiones básicas por ser donde se tejen las relaciones sociales, los vínculos, la pertenencia, la convivencia, etc., que van a posibilitar la mayor o menor cercanía y contactos entre 
la sociedad receptora, los agentes sociales, las instituciones y los distintos colectivos de personas inmigrantes y solicitantes de asilo que, en definitiva, pueden facilitar o dificultar la integración social a medio y largo plazo.

Ahora bien, el acceso a la vivienda presenta, por su parte, dos dificultades fundamentales. En primer lugar, el elevado precio del alquiler, cuyo afrontamiento se hace especialmente complicado con la precaria incorporación laboral lograda en la mayoría de los casos, tal y como se ha comentado anteriormente. Y, en segundo lugar, los numerosos recelos que la población autóctona muestra a la hora de alquilar una vivienda a la población inmigrante, sea cual sea su situación administrativa en este territorio (Vicente, 2009).

Hasta este momento, los mayores esfuerzos públicos en materia residencial se han hecho en el sentido de aumentar el parque de viviendas municipal, foral o autonómico destinado a la fase de acogida. Ahora bien, algunas voces ya apuntan a que quizá habría que hacer un replanteamiento de esta situación, ya que la fase de acogida es financiada por el Ministerio, mientras que en la fase de integración y de autonomía, estas personas dependen en mayor medida de sus escasos recursos, al tiempo que no se ofrecen otros apoyos residenciales que los de urgencia. Una realidad que, en cierta medida, va a tratar de paliar el programa Goihabe de la Diputación Foral de Bizkaia, aunque todavía con bastantes limitaciones a día de hoy:

Igual lo que tenemos que cambiar nosotros también es el chip, es que los recursos que cedamos se dirijan más a esa segunda fase y a la fase de autonomía, porque es donde de verdad se están detectando esas necesidades de vivienda. (Representante del Departamento de Empleo y Políticas Sociales, Gobierno Vasco)

Por otro lado, dentro del proceso de integración social, las personas que han visto reconocida su condición de asilada, al igual que quienes la han visto denegada, tienen abierta la puerta de los servicios sociales, si bien para ello van a tener que cumplir las mismas condiciones impuestas al conjunto de la población. Unas condiciones que no siempre van a poder satisfacer desde el mismo momento en que se quedan sin el soporte económico del Ministerio, recibido a través de las organizaciones conveniadas con este fin:

No hay una vía específica de acceso, por ejemplo, a los servicios sociales para estas personas solicitantes de asilo. Hay un 
tratamiento igualitario con cualquier otra persona. (...) El reto está sobre todo en las siguientes fases y en cómo poder asegurar que no haya vacíos de atención entre la atención plena de los seis primeros meses y el siguiente salto hasta los tres años para que, como cualquier otra persona, puedan tener la cobertura de la RGI [Renta de Garantía de Ingresos]. Para eso tenemos, por lo menos a nivel municipal, unas competencias limitadas. (Personal técnico municipal de inmigración)

Las personas solicitantes de asilo van a poder disfrutar, desde la primera renovación de su tarjeta, de un permiso de trabajo. Sin embargo, dada la actual coyuntura económica y de empleo, este permiso no va a garantizar que encuentren un trabajo remunerado con el que cubrir sus necesidades básicas, tal y como se ha detallado anteriormente. Y, por otra parte, quienes vean su solicitud de asilo denegada van a perder, asimismo, dicha autorización para desempeñar un empleo regular, quedando sus opciones laborales reducidas a la economía sumergida. Sin embargo, al menos durante una primera etapa, su acceso a los servicios sociales también va a quedar fuertemente limitada a las ayudas de emergencia (AES), ya que para poder solicitar la Renta de Garantía de Ingresos es preciso poder demostrar que la persona lleva ya al menos tres años empadronada en la CAPV:

Lo bueno que tienen los peticionarios de asilo es que pueden trabajar. Pero es que, claro, si no hay trabajo se quedan fuera del sistema de protección social porque no llevan tres años empadronados. (...) La RGI no tiene ninguna exención para peticionarios de asilo. [...] Entonces, ¿qué pasa? Que te llega al Servicio Social de Base una familia con toda la dureza de un proceso de asilo, que estaba acogida en un centro temporal, y le dicen: "se te acabó. (...) En los Servicios Sociales de Base se pueden tramitar unas ayudas de emergencia que llegan para lo que llegan y ya está. Si no hay un empleo, no hay una continuidad. (Personal técnico municipal de inmigración)

Ahora bien, las ayudas de emergencia, que exigen un periodo de empadronamiento mucho menor que la RGI —seis meses-, son subvenciones puntuales, dependientes de la disponibilidad de fondos, que pueden contribuir a aliviar una situación precaria de forma puntual, aunque poco tienen que ver con la renta de garantía de ingresos: 
Para las AES tienen seis meses de empadronamiento. Pero las AES es una subvención para financiar alimentación, alojamiento, tal. [...] No es una prestación no contributiva, como la RGI, que es un sueldo. Esto es una subvención para familias en situación de emergencia que cumpla los requisitos que, además, nos los estipula el Gobierno Vasco. (Personal técnico municipal de inmigración)

Además de estas ayudas públicas económicas ocasionales, a nivel municipal se ofrecen otras igualmente con carácter puntual, en materia residencial, por ejemplo, y que también han sido valoradas en las entrevistas mantenidas como insuficientes para aliviar la situación de precariedad de aquella población solicitante de asilo a la que todavía le falta un periodo considerable para acceder a la RGI. Por ello, no son las instituciones públicas las únicas que tratan de ayudar a estas y otras personas en situación vulnerable en el afrontamiento de estas necesidades perentorias, bien sea a través de ayudas económicas, de la aportación de información, del ofrecimiento de pisos de acogida, o articulando la solidaridad ciudadana, manifestada en el acogimiento familiar durante breves periodos de tiempo. Con todo, estos apoyos son calificados frecuentemente como insuficientes, por lo que se plantea la necesidad de hacer una profunda reflexión, cada institución desde su ámbito competencial, con el objeto de garantizar una respuesta más acorde a las necesidades específicas de las personas solicitantes de asilo, de modo que no se produzcan estos saltos tan abruptos en su protección.

\section{Mecanismos de coordinación específicos: la puesta en marcha del Programa Goihabe}

Ante la constatación de la necesidad de un mayor apoyo por parte de la población solicitante de asilo y refugiada tras la finalización de la etapa de acogida, la Diputación Foral de Bizkaia ha puesto en marcha el programa Goihabe en el año 2016. Se trata de un programa piloto vinculado al proyecto político de la Diputada de Empleo, Inclusión Social e Igualdad, dentro del marco de un acuerdo de gobierno, y centrado en la fase de integración de las personas solicitantes de asilo y de las personas refugiadas. Su objetivo es dar una respuesta integral a sus necesidades de incorporación social, evitando situaciones de marginalidad. Y para ello se va a articular 
a través de la figura de la subvención a entidades de iniciativa social sin ánimo de lucro con una amplia trayectoria en labores de defensa de los Derechos Humanos, la integración y la promoción del desarrollo integral de las personas refugiadas y migrantes con necesidad de protección internacional o en riesgo de exclusión, y con una estructura adecuada y suficiente para atender a esta situación. Más concretamente, las cuatro entidades con las que la Diputación Foral de Bizkaia ha firmado un convenio con el objeto de desarrollar el programa Goihabe son: la Asociación CEAR-Euskadi / Comisión de Ayuda al Refugiado, Cruz Roja Española, Cáritas Diocesana de Bilbao y la Fundación Ellacuría.

Estas cuatro organizaciones se comprometen a desarrollar acciones en los ámbitos de: a) la intervención socioeducativa, psicosocial y afectivo emocional; b) la información y orientación sobre derechos y deberes de las personas extranjeras, procedimiento de asilo en España, retorno voluntario a sus países de origen, sobre el programa y los servicios públicos y privados, etc.; c) el apoyo sociolingüístico (idioma, traducción e interpretación), d) plan formativo-laboral; e) la asistencia jurídica en relación con el procedimiento de protección internacional y otras consultas relacionadas con la extranjería; f) las prestaciones económicas (apoyo económico básico, para el caso de necesidad vital, condicionado al desarrollo de un programa/ itinerario de integración, g) el apoyo residencial o, h) la mediación, intermediación y sensibilización. Ahora bien, el objetivo no es duplicar esfuerzos ni entrar en una competencia por las personas objeto del programa, por lo que no se plantea su reparto entre las cuatro instituciones implicadas sino una distribución de competencias, en función de aquellos ámbitos en los que vienen trabajando y acumulando más experiencia cada una de ellas.

La población destinataria del programa Goihabe es aquella que ha solicitado asilo y también aquella que, una vez reconocida la protección internacional, precise de apoyo para lograr su plena incorporación a la sociedad de acogida. Ahora bien, es preciso que dicha persona haya contado con el apoyo del Ministerio, a través de alguna de las instituciones conveniadas a tal fin, al menos tres meses antes de su solicitud de incorporación a este programa. No importa, sin embargo, cuál sea su situación administrativa en el momento de incorporarse a Goihabe: podría seguir a la espera de que se resuelva su solicitud de asilo, podría disfrutar ya del reconocimiento de protección internacional o, incluso, podría haber visto su solicitud 
denegada. Y ello porque el objetivo es precisamente apoyar a este colectivo en su proceso de inclusión en la sociedad de acogida. Apoyo que podrá extenderse durante el tiempo necesario, con un periodo máximo de tres años (no olvidemos que con tres años de empadronamiento estas personas también pueden acceder a la RGI, si cumplen el resto de condiciones exigidas para ello).

Nuestro objetivo no está en función de su tramitación administrativa o de las resoluciones de la Oficina de Asilo. Nuestros parámetros son de inclusión social, no de inclusión por la residencia legal o de la consecución de tarjetas de residencia. (...) [Denegada la solicitud de asilo, ¿continúa en el programa?] ¡Sí! (Representante del Departamento de Empleo, Inclusión Social e Igualdad, Diputación Foral de Bizkaia)

El programa Goihabe, se ha articulado a través de la figura de la subvención, y ha sido dotado con un importe total de $242.000 €$, con cargo al ejercicio del 2016, distribuidos de la siguiente manera: Cruz Roja Española: 100.000 €; Asociación CEAR-Euskadi \& Comisión de Ayuda al Refugiado, $61.000 €$; Cáritas Diocesana de Bilbao, $61.000 €$ y Fundación Social Ignacio Ellacuría, $20.000 €$.

Su reciente puesta en marcha todavía impide hacer una evaluación exhaustiva de su funcionamiento, pero tanto la Diputación Foral de Bizkaia como las organizaciones encargadas de su desarrollo subrayan la necesidad de profundizar en los recursos destinados a la atención de la necesidad extraordinaria y urgente de integración de las personas solicitantes de asilo y beneficiarias de protección internacional, una vez superada la etapa de acogida financiada por el Ministerio de Interior. Y para ello reivindican una mayor solidaridad y una mayor coordinación interinstitucional, no sólo entre las partes actualmente implicadas, sino también con otras que podrían incorporarse en un futuro próximo.

\section{REFLEXIONES FINALES}

La crisis europea del sistema de asilo no solo ha puesto en evidencia la necesidad de coordinar mayormente en términos cualitativos los procesos de integración y acogida, sino que también insta a actualizar los planes de contingencia tanto a nivel nacional como local para garantizar una capacidad real de acogida suficiente y adecuada. 
Un primer paso en la optimización de los procesos desde el punto de vista jurídico es la transposición del denominador común previsto en la Directiva 2011/33/UE que en España, a falta del necesario desarrollo reglamentario, podría contribuir a mejorar la coordinación entre las administraciones competentes en los procesos de acogida e integración así como en la resolución de los expedientes de solicitud de asilo ante situaciones de especial vulnerabilidad.

De igual modo, un segundo paso para invertir las lógicas de las velocidades existentes, es revisar de forma crítica los términos de la acogida integral e integración del SAI y reflexionar en qué medida estos pueden consolidar un sistema asistencialista, cortoplacista y rígido. Un sistema que no solo implica un incremento cuantitativo de las plazas sino una mejora cualitativa de las condiciones de vida ofrecidas a las personas solicitantes de protección internacional, con independencia del lugar de llegada al territorio. Máxime si las sinergias generadas por la compartimentación del propio sistema puede llegar a forzar a que muchas personas sean enviadas a un centro que difícilmente se adecua a sus necesidades o a lugares donde encuentran grandes dificultades para encontrar salidas laborales, redes de apoyo, cuando no se identifican correctamente las diferentes situaciones de vulnerabilidad ni se conviene la adaptación de los procesos a las propias trayectorias de las personas solicitantes de protección internacional. Una muestra de ello, como se ha contrastado en las entrevistas, es el itinerario de integración por fases diferenciadas en función de la existencia de una situación de vulnerabilidad o no (18 meses o 24 meses), que no se corresponde al proceso de integración real dado que éste viene marcado frecuentemente por el retraso o por la denegación del reconocimiento del estatuto de persona beneficiaria de protección internacional.

Por último, como tercer paso dentro del endeble tránsito entre la fase de integración y autonomía, por el momento, si bien no existe una previsión explícita de medidas de atención específicas de la población refugiada, Euskadi se encuentra entre las Comunidades Autónomas que optan por integrarla en programas generales de inserción social para población migrante; aunque ello implica mejorar los mecanismos de coordinación y comunicación institucional entre municipios, EUDEL, diputaciones, departamentos del Gobierno Vasco y las diferentes entidades del Tercer Sector. 
En particular, este sería el objetivo en Bizkaia del Programa Goihabe: pese a su breve recorrido, refuerza y respalda la eficacia de las fases de integración y autonomía del SAI, en las que la población solicitante de protección internacional se puede ver más expuesta a situaciones de exclusión social al no poder cubrir las necesidades básicas por falta de ayudas o redes sociales. La coordinación inter- e intra-institucional en el Territorio Histórico de Bizkaia ha dado una respuesta a la mayor sensibilización generada por la crisis migratoria, pero en esta primera fase embrionaria del proceso se requiere reforzar la información y la previsión de itinerarios de formación específica. Sin duda, el aumento y la mejora de los niveles de sensibilización y de formación especializada en esta materia dirigidos al personal de la administración pública a nivel general, y de Bizkaia en particular, no sólo harían más operativos los procesos sino que permitirían identificar las necesidades especiales de la población refugiada desde su llegada hasta su inserción en la sociedad, atendiendo a las necesidades especiales que, lejos de ser situaciones de emergencia o coyunturales, van a ser un continuum no exento de variables.

\section{REFERENCIAS BIBLIOGRÁFICAS}

AIDA (2016): Wrong counts and closing doors. The reception of refugees and asylum seekers in Europe. Brussels: ECRE, Recuperado de https://goo.gl/ qr79pC

AMNISTÍA INTERNACIONAL (2016): El asilo en España. Un sistema de acogida poco acogedor. Madrid: Amnistía Internacional, Recuperado de https://goo.gl/Y1WcXx

Arando, J., Mahia, R., Moya, D. y Sánchez Montijano, E. (2016): El año de los refugiados. Anuario CIDOB de la Inmigración 2015-2016 (nueva época). Barcelona: Bellaterra.

Arnold-Fernández, E. E. y Stewart, P. (2013): El derecho de los refugiados al trabajo, Revista de Migraciones Forzadas. 44, 92-93. Recuperado de http://www.fmreview.org/es/detencion/arnoldfernandez-pollock.pdf.

BauböcK, R. y TRIPKovic, M. (2017): The integration of migrants and refugees. an EUI forum on migration, citizenship and demography. Florence: European University Institute, Robert Schuman Centre for Advanced Studies.

Blanco, C. (1990): La integración de los inmigrantes en Bilbao. Bilbao: Ayuntamiento de Bilbao.

Carrera, S., Blockmans, S., Gros, D. y GuILD, E. (2015): The EU's response to the refugee crisis. Taking stock and setting policy priorities. CEPS Essays. Bruselas: CEPS. Recuperado de http://aei.pitt.edu/70408/ 
CEAR (2016): Informe 2016: Las personas refugiadas en España y Europa. Madrid: CEAR. Recuperado de http://www.cear.es/wp-content/ uploads/2016/06/Informe_CEAR_2016.pdf

De Lucas, J. (2015): Mediterráneo: El naufragio de Europa. Valencia: Tirant Lo BLANCH.

Defensor del Pueblo (2016): Estudio sobre el asilo en España. La protección internacional y los recursos del sistema de acogida. Madrid: Defensor del Pueblo. Recuperado de https://www.defensordelpueblo.es/wp-content/ uploads/2016/07/Asilo_en_Espa\%C3\%B1a_2016.pdf

EUROPEAN PARLIAMENT (2013): Comparative study on the best practices for the integration of resettled refugees in the EU Member States. ECRE, Brussels: Study commissioned by the Policy Department C: Citizens' Rights and Constitutional Affairs.

EUROPEAN PARLIAMENT (2016): Labour market integration of refugees: Strategies and good practices, Directorate General for internal policies. Policy Department A: Economic and scientific policy. Bruselas: European Parliament, IP/A/EMPL/2016-08. Recuperado de http://www.europarl.europa. eu/RegData/etudes/STUD/2016/578956/IPOL_STU(2016)578956_EN.pdf

EMN (2015): Integration of beneficiaries of international/humanitarian protection into the labour market: policies and good practices. Bruselas: European Migration Network.

FERnÁNDEz SÁnchez, P. A. (2015): Las políticas públicas de integración social de los refugiados, como obligación internacional y europea. En M. ABAD, M. C. Barranco y M. C. Llamazares (coord.) Derecho y minorías (pp. 163194). Madrid: Dykinson.

FIELDEN, A. (2008): Local integration an under-reported solution to protracted refugee situations. Research paper No. 118, UNCHR.

Foro PARA LA INTEGRACIÓN SOCIAL DE LOS INMIGRANTES (2015). Informe sobre la situación de la integración de los inmigrantes y refugiados en España. Madrid: FSI.

García Mahamut, R. y Galparsoro, J. (2010): Régimen jurídico del derecho de asilo en la Ley 12/2009. Madrid: Centro de Estudios Políticos y Constitucionales.

Giménez, C. (2003): Pluralismo, multiculturalismo e interculturalidad: propuesta de clarificación y apuntes educativos. Educación y Futuro: Revista de Investigación Aplicada y Experiencias Educativas, 8, 9-26.

Iglesias, J., Urrutia, G., Buades, J., Estrada, C. y Vicente, T. (2018): ¿Acoger sin integrar? El Sistema de Acogida y las condiciones de integración de la población refugiada en España. Sevilla: S. J. M.

IgLEsias, J. y EstradA, C. (2018): ¿Birds of passage? La integración social de la población refugiada en España. Iberoamerican Journal of Development Studies, 7 (1). Recuperado de http://ried.unizar.es/public/abstracts/birdsofpassage.pdf

Joppke, Ch. (2007): Transformation of immigrant integration in Western Europe: Civic integration and antidiscrimination in the Netherlands, 
France, and Germany. World Politics, 59(2), 243-273. Recuperado de http://policyoptions.irpp.org/wp-content/uploads/2014/08/joppke.pdf

Koopmans, R. (2010): Trade-offs between equality and difference: Immigrant, integration, multiculturalism and the Welfare State in cross-national perspective. Journal of Ethnic and Migration Studies, 36 (1), 1-26. DOI: $10.1080 / 13691830903250881$

LA SPINA, E. (2017): La protección de la unidad familiar en contextos de crisis migratoria: la historia de dos casos. Universitas: Revista de filosofía, derecho y política, 25, 163-186. Recuperado de https://e-revistas.uc3m.es/ index.php/UNIV/article/view/3561/2176

La Spina, E., Vicente, T., Urrutia, G. y Morondo, D. (2017): La población refugiada en Bizkaia y su proceso de integración. Bilbao: Diputación Foral de Bizkaia. Recuperado de https://goo.gl/e8k9bJ

MEYSS-SGII (2016): Sistema de acogida e integración para solicitantes y beneficiarios de protección internacional. Manual de gestión. Madrid: MEYSS.

Moraes, N. y Romero, H. (2016): La crisis de los refugiados y los deberes de Europa. Madrid: Los Libros de la Catarata.

NAïr, S. (2016): Refugiados. Madrid: Ed. Crítica.

OCDE (2016): Making integration work. Refugees and others in need of protection, Paris: OCDE Publishing.

OfFE, K. (2016): For a robust policy of integration of refugees. Social Europe online. Recuperado de https://www.socialeurope.eu/2016/03/robust-policy-integration-refugees/

Pascouau, Y. (2014): Measures and rules developed in the EU Member States regarding integration of third county nationals. Comparative Report. Bruselas: European Policy Centre. Recuperado de https://ec.europa.eu/ migrant-integration/librarydoc/integration-measures-in-the-euupdateddecember-2014

Peers, S., Moreno-Lax, V., Garlick, M. y Guild, E. (eds.) (2015): EU immigration and asylum law (text and commentary), second revised edition, Vol. 3 Asylum Law, Brill Nijhof.

Pumares, P. (1998): ¿Qué es la integración? Reflexiones sobre el concepto de integración de los inmigrantes. En CHECA, F. (eds). Africanos en la otra orilla. Trabajo cultura e integración en la España mediterránea (pp. 289318). Barcelona: Icaria.

Scholten, P. y Penninx, R. (2015): The Multilevel Governance of Migration and Integration. En B. Garcés-Mascareñas y R. PEnninx (eds.). Integration process and policies in Europe. IMISCOE Research (pp. 91-108). Dordrecht: Springer.

Tsourdi, E. (2015): Reception conditions for asylum seekers in the EU: towards the prevalence of human dignity. Journal of Immigration, Asylum and Nationality Law, 29, (1), 9-24.

UNCHR (2013): A new beginning. Refugee integration in Europe. Geneva: UNCHR. Recuperado de http://www.refworld.org/pdfid/522980604.pdf 
Vicente, T. L. (2008): La escuela vasca ante la realidad de la inmigración: un nuevo desafío. Vitoria-Gasteiz: Gobierno Vasco

Vicente, T. L. (2009): La vivienda: ¿Un derecho humano o un factor de exclusión de la población inmigrante? En G. Urrutia (ed.): Derechos Humanos $y$ discriminación. ¿Nuevos o continuos retos? (pp. 129-157). Zarautz: Alberdania. 